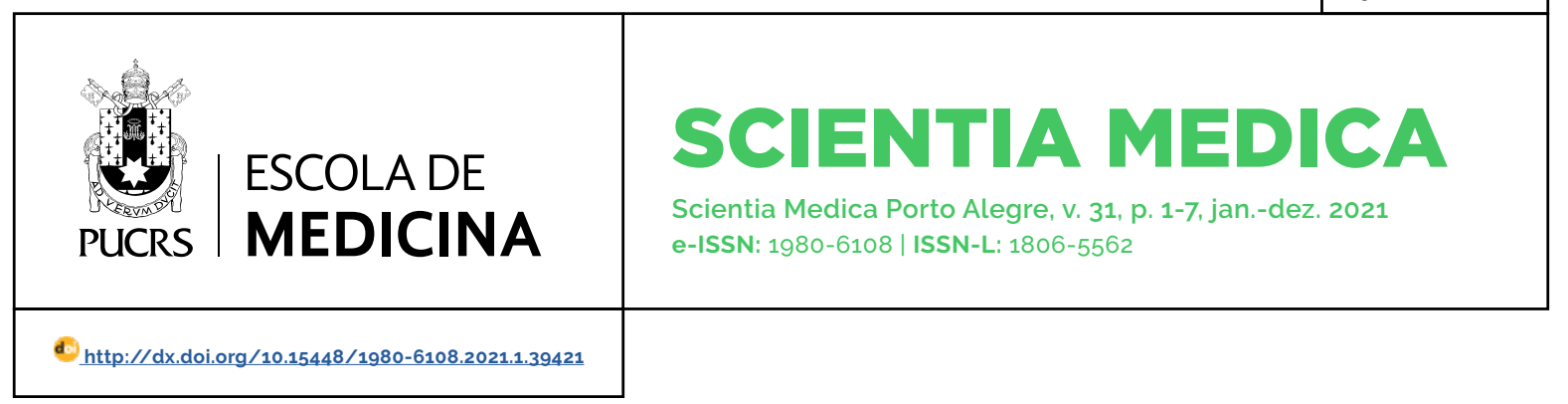

ARTIGO ORIGINAL

\title{
Comparação epidemiológica entre as fraturas de fêmur proximal atendidas em um hospital secundário e um terciário
}

\author{
Epidemiological comparison between proximal femur fractures in a secondary and a \\ tertiary hospital
}

\author{
Weby Delsin Mizael $^{1}$ \\ orcid.org/0000-0002-8805-7022 \\ weby_dm@hotmail.com
}

Leandro Campos Vilela ${ }^{1}$

orcid.org/0000-0003-4953-2908

leandrovilela1@hotmail.com

\section{Ronaldo Parissi \\ Buainain $^{1}$}

orcid.org/0000-0002-3465-5254

ronaldopb1975@gmail.com

\section{Nilson Nonose ${ }^{1}$}

orcid.org/0000-0002-5397-2271 ninonose@yahoo.com.br

Recebido em: 26 out. 2020. Aprovado em: 27 jul. 2021. Publicado em: 17 set. 2021

\section{Resumo}

Objetivo: comparar a ocorrência de fraturas de fêmur proximal atendidas em um hospital secundário e em um terciário.

Método: a amostra deste estudo foi composta por todos os pacientes que apresentaram fratura de fêmur proximal atendidos em dois serviços hospitalares, no período de 2015 a 2017, sendo realizada a avaliação dos prontuários.

Resultados: embora um maior número de cirurgias tenha sido realizado no hospital terciário, os pacientes atendidos no hospital secundário apresentaram maior número de complicações, provavelmente em decorrência de mais tempo de espera pelo procedimento, incluindo as cirurgias que precisaram ser remarcadas. É provável, também, que esse fato tenha implicado em maior tempo de internação e necessidade de encaminhamento para UTI no periodo pós-operatório imediato, fenômenos também observados no hospital secundário.

Conclusão: estes resultados chamam atenção para a necessidade de reformulação dos protocolos de atendimento a pacientes com fratura proximal de fêmur, visando sanar os problemas aqui apresentados.

Palavras-chave: ortopedia, traumatologia, epidemiologia, fêmur, fraturas.

\section{Abstract}

Aim: to compare the occurrence of femur fractures treated in a secondary and in a tertiary hospitals.

Method: the sample of this study consisted of all patients who had fractures of the proximal femur treated at two hospital services, from 2015 to 2017, and the medical records were evaluated.

Results: a greater number of surgeries has been performed at the tertiary hospital, although the secondary hospital patients had the highest number of complications, resulted probably by longer waiting times for the procedure, including surgeries that needed to be scheduled. It is also likely that this fact implied in a longer hospital stay and the need for referral to the Intensive Unit Care in the immediate postoperative period, also observed in secondary hospitals.

Conclusion: these results call attention to the need to reformulate the protocols for the care of patients with proximal fracture of the femur, to solve the problems presented here.

Keywords: orthopedics, traumatology, epidemiology, femur, fractures. 


\section{Introdução}

As fraturas do fêmur proximal representam um importante tópico no campo da cirurgia ortopédica devido às suas proporções econômicas, assim como em relação à morbimortalidade. A taxa de incidência geral de fraturas do fêmur proximal é de, aproximadamente, 23 para cada 10 mil pacientes (1), sendo divididas principalmente em três tipos: transtrocantéricas, que ocorrem em, aproximadamente, $57 \%$ dos casos; as fraturas de colo do fêmur, que acometem cerca de $42 \%$ dos pacientes (2); e as fraturas na região subtrocantérica, que acometem, aproximadamente, 5 a 10\% (1). Os dois tipos com maior acometimento são as fraturas trocantéricas e as fraturas do colo. $O$ primeiro tipo (trocantéricas) apresenta um curso mais grave, com maior perda de sangue, taxa de mortalidade mais alta, acometendo pacientes mais velhos, quando comparadas ao segundo tipo (colo femoral) e, geralmente, mais complicações (3).

Além do dano econômico e social, as fraturas proximais do fêmur têm um impacto negativo na qualidade de vida dos pacientes e causam uma alta taxa de mortalidade por complicações pós-fratura ou pós-operatórias. Outro ponto importante é que, no Brasil, o número de pessoas com o diagnóstico de osteoporose chega a 10 milhões, e os gastos com o tratamento e a assistência no Sistema Único de Saúde são altos. Só em 2010, o Sistema Único de Saúde gastou, aproximadamente, R\$ 81 milhões para a atenção ao paciente portador de osteoporose e vítima de quedas e fraturas (4). Em meados da década de 2010 já se estimava que, mundialmente, as fraturas de fêmur em idosos contribuiriam para um gasto público de, aproximadamente, 131 bilhões de dólares (5). Dados mais recentes apontaram, no ano de 2019, a ocorrência de 397.585 mil internações no Brasil devido a fraturas de fêmur somente no Sistema Único de Saúde, que geraram um gasto de $\mathrm{R} \$$ 85.839.680,38 (6).

A mortalidade anual em idosos devido às quedas apresenta um pico por volta dos 85 anos e, somente cerca da metade dos idosos admitidos em um hospital após esse acidente, permanece viva no ano seguinte (7). Fica evidente que a demanda de pacientes idosos com trauma de fêmur é maior que a oferta fornecida nos hospitais públicos, e essa é a realidade em saúde no Brasil, portanto se faz necessário melhorar a qualidade da assistência prestada para essa população (8).

Ainda, entende-se a necessidade de reflexão sobre a importância de se realizar levantamentos epidemiológicos pontuais relacionados à ocorrência de fraturas proximais de fêmur, atendidas nos serviços de ortopedia e traumatologia. Tais levantamentos permitem conhecer o perfil específico do paciente acometido, o que, por sua vez, pode servir de base para o estabelecimento de estratégias visando sistematizar o atendimento a esses pacientes, buscando reduzir, por exemplo, o tempo de espera pela cirurgia, reduzir os dias improdutivos, e melhorar o prognóstico dos pacientes.

A proposta do presente estudo é comparar o perfil epidemiológico dos pacientes com fraturas de fêmur proximal atendidos em dois hospitais, sendo um secundário e outro terciário, considerando uma série de parâmetros relacionados a esses pacientes, incluindo suas caracteristicas, assim como fatores relacionadas ao serviço no qual cada paciente foi atendido.

\section{Método}

Trata-se um estudo descritivo, retrospectivo, de abordagem quantitativa, realizado no Serviço de Ortopedia e Traumatologia do Hospital Universitário São Francisco na Providência de Deus, um hospital terciário, localizado na cidade de Bragança Paulista, SP e no Hospital Santa Casa Anna Cintra, um hospital secundário, localizado na cidade de Amparo, SP. O Hospital Universitário São Francisco na Providência de Deus, classificado como hospital terciário, utiliza-se de recursos tecnológicos mais avançados, possui maior corpo clínico e mais capacidade para realização de diagnósticos e tratamentos. Já o Anna Cintra, classificado como hospital secundário, possui um corpo clínico menor, além de reduzida disponibilidade de serviços complementares, quando comparado ao serviço terciário ( 9 ).

A amostra deste estudo foi composta por todos os pacientes que apresentaram fratura de fêmur proximal atendidos em ambos os 
serviços, no periodo de 2015 a 2017, visto que apenas os prontuários dos pacientes atendidos neste intervalo de tempo estavam disponiveis para avaliação em ambos os hospitais. Desses pacientes, foram avaliadas as seguintes variáveis: idade, sexo, lateralidade, tipo de fratura, comorbidades associadas à fratura, município de origem, mecanismo do trauma, possiveis intercorrências operatórias, dias de hospitalização, classificação American Society of Anesthesiologists, necessidade de transferência para a Unidade de Terapia Intensiva (UTI) no período pós-operatório, possíveis complicações e se a cirurgia precisou ser suspensa, incluindo o motivo da suspensão e o número de ocorrências.

As variáveis foram apresentadas de forma descritiva, com base em sua frequência absoluta seguida de sua respectiva frequência relativa ao tamanho total da amostra. O teste de comparação entre duas proporções, realizado no software Microsoft Excel $2010^{\circledR}$, foi utilizado para verificar diferenças estatisticamente significantes entre as frequências observadas para cada variável, considerando um nivel de significância de 95\%.

Este trabalho foi aprovado pelo comitê de ética em pesquisa da Universidade de São Francisco, SP, segundo parecer consubstanciado número 3.941.915, de 30 de março de 2020.

\section{Resultados}

A amostra deste estudo foi constituida por 367 pacientes com fratura de fêmur proximal. Desses, 137 (37\%) foram atendidos no hospital secundário, enquanto 230 (63\%) foram atendidos no hospital terciário. A Tabela 1 traz o resumo das comparações entre as variáveis mais representativas, considerando a sua frequência, observadas nos dois hospitais avaliados. No hospital secundário, 92 (67\%) das fraturas ocorreram em individuos do sexo feminino e no terciário, 131 (57\%) eram mulheres $(p=0,15)$.
TABELA 1 - Comparação entre as variáveis observadas no hospital secundário e terciário.

\begin{tabular}{|c|c|c|c|}
\hline Variável avaliada & $\begin{array}{l}\text { Hospital } \\
\text { secundário } \\
n=137\end{array}$ & $\begin{array}{l}\text { Hospital } \\
\text { terciário } \\
n=230\end{array}$ & $\mathrm{p}$ \\
\hline $\begin{array}{l}\text { Origem na cidade do } \\
\text { hospital }\end{array}$ & $87(64)$ & $71(31)$ & 0,001 \\
\hline Sexo feminino & $92(67)$ & $131(57)$ & 0,15 \\
\hline $\begin{array}{l}\text { Idade acima de } 80 \\
\text { anos }\end{array}$ & $64(46)$ & $74(32)$ & 0,001 \\
\hline Etnia caucasiana & $110(80)$ & $167(73)$ & 0,20 \\
\hline Fratura do tipo fechada & 136(99) & 227(99) & 0,93 \\
\hline $\begin{array}{l}\text { Localização } \\
\text { transtrocanteriana }\end{array}$ & $61(45)$ & $116(50)$ & 0,21 \\
\hline Lateralidade esquerda & $69(50)$ & 119(52) & 0,77 \\
\hline $\begin{array}{l}\text { Presença de fratura } \\
\text { associada }\end{array}$ & $12(9)$ & 28(12) & 0,09 \\
\hline $\begin{array}{l}\text { Mecanismo do trauma } \\
\text { (queda da própria } \\
\text { altura) }\end{array}$ & $118(86)$ & $179(78)$ & 0,18 \\
\hline $\begin{array}{l}\text { Presença de } \\
\text { comorbidades }\end{array}$ & 126(92) & 196(85) & 0,31 \\
\hline Tabagismo & $37(27)$ & $51(22)$ & 0,08 \\
\hline Etilismo & $11(8)$ & $15(7)$ & 0,29 \\
\hline Uso de medicamentos & $120(88)$ & 186(81) & 0,30 \\
\hline $\begin{array}{l}\text { Tempo de espera } \\
\text { acima de } 96 \mathrm{~h}\end{array}$ & $70(51)$ & $43(18)$ & 0,001 \\
\hline Classificação ASA III & $66(48)$ & $109(47)$ & 0,86 \\
\hline $\begin{array}{l}\text { Adiamento por falta de } \\
\text { vaga na UTI }\end{array}$ & $35(25)$ & $35(15)$ & 0,001 \\
\hline Raquianestesia & $119(87)$ & $130(56)$ & 0,001 \\
\hline $\begin{array}{l}\text { Utilização de haste no } \\
\text { reparo }\end{array}$ & $46(34)$ & $70(30)$ & 0,35 \\
\hline Indicação para UTI & 100(73) & $70(30)$ & 0,001 \\
\hline Complicações & $43(31)$ & $56(24)$ & 0,01 \\
\hline $\begin{array}{l}\text { Tempo de internação } \\
\text { acima de cinco dias }\end{array}$ & $112(81)$ & $107(47)$ & 0,001 \\
\hline Letalidade & $6(4)$ & $17(7)$ & 0,05 \\
\hline
\end{tabular}

ASA, American Society of Anesthesiologists; UTI, Unidade de Terapia Intensiva; Dados apresentados em frequência absoluta e relativa ao tamanho da amostra. 
Na avaliação do tempo de espera para realização da cirurgia, inicialmente no hospital secundário, em 19 (14\%) pacientes o procedimento foi realizado entre 24 e 48 horas após o trauma, em 48 (35\%) foi realizado com espera de 48 até 96 horas, e em 70 (51\%) pacientes a espera pela cirurgia ultrapassou as 96 horas. No hospital terciário, 119 (52\%) pacientes esperaram entre $24 \mathrm{e}$ 48 horas, 68 (30\%) esperaram de 48 e 96 horas, e 43 (18\%) esperaram mais de 96 horas.

Das 137 cirurgias agendadas no hospital secundário, em 102 (75\%) não houve suspensão do procedimento por falta de vaga na UTI. Das 35 restantes, 25 (18\%) foram suspensas uma vez, 6 (4\%) foram suspensas duas vezes, e 4 (3\%) foram suspensas três vezes (correspondendo, respectivamente a $71 \%, 17 \%$ e $12 \%$ das cirurgias suspensas). No hospital secundário, das 230 cirurgias, 195 $(84,5 \%)$ ocorreram na data originalmente progra- mada. Das 35 cirurgias suspensas, 21 (9\%) foram suspensas uma vez, 13 (6\%) foram suspensas duas vezes, e 1 (0.5\%) foi suspensa três vezes (correspondendo, respectivamente a 60\%, 37\% e 3\% das cirurgias suspensas). Os motivos para suspensão das cirurgias incluíram a indisponibilidade de vaga para internação na UTI em caso de necessidade, além de instabilidade clínica dos pacientes.

Quando verificado se houve indicação para internação em UTI no pós-operatório imediato no hospital secundário, o encaminhamento foi necessário em 100 (73\%) pacientes e, no hospital terciário, 70 pacientes (30\%) foram indicados para UTI ( $p=0,001)$.

Do total, 43 (31\%) pacientes operados no hospital secundário apresentaram complicações e nos pacientes operados no hospital terciário, estas foram identificadas em 56 (24\%) casos $(p=0,01)$. A distribuição das complicações está representada na Figura 1

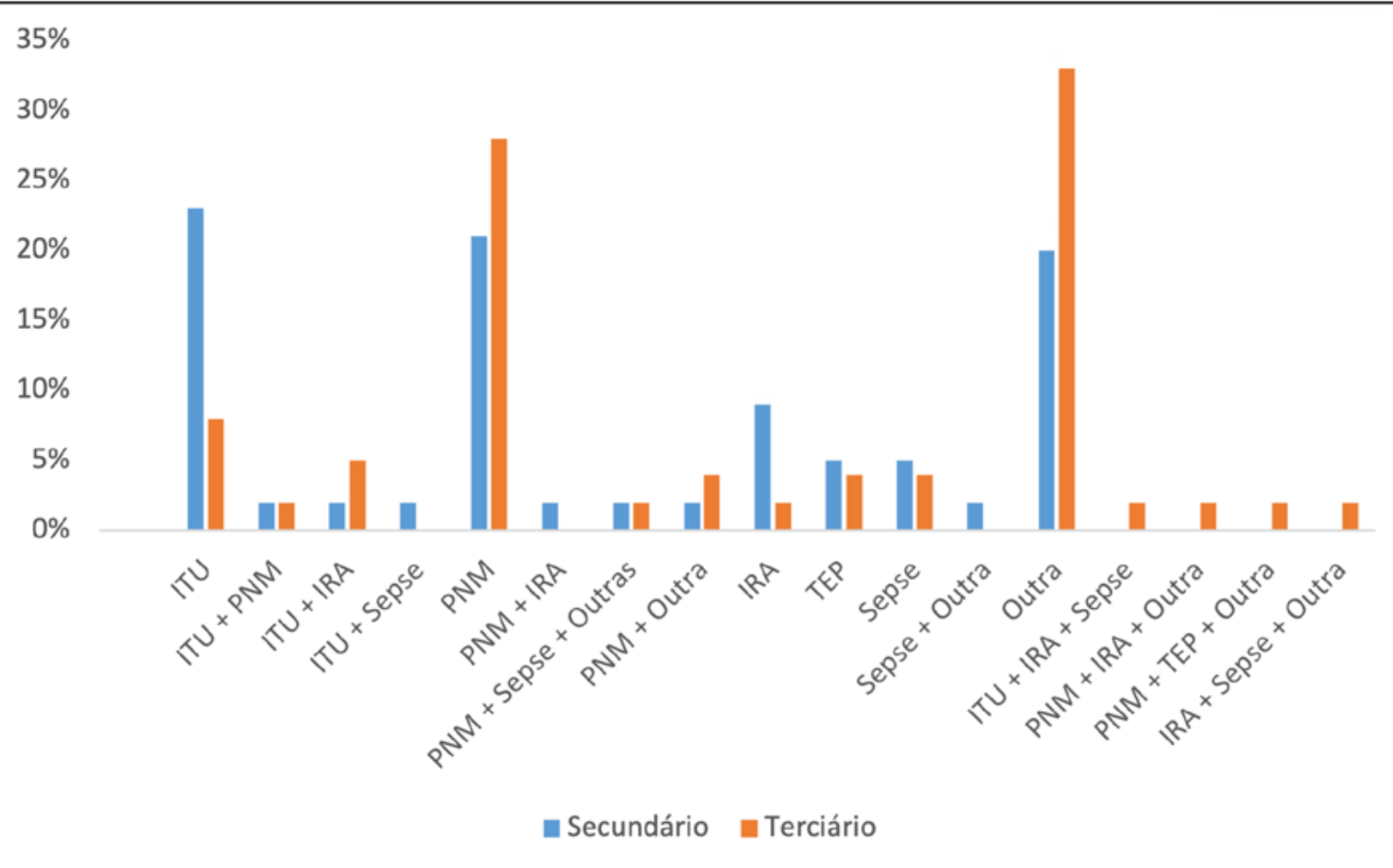

Figura 1 - Distribuição percentual de casos pelas complicações pós-operatórias no hospital terciário, que se se utiliza de recursos tecnológicos mais avançados, possui maior corpo clínico e maior capacidade para realização de diagnósticos e tratamentos, e no hospital secundário, que possui um corpo clínico menor, além de reduzida disponibilidade de serviços complementares.

IRA, insuficiência renal aguda; ITU, infecção do trato urinário, PNM, pneumonia; TEP, tromboembolia pulmonar. 
Na avaliação do tempo de internação dos pacientes operados no hospital secundário, este foi menor do que três dias em 5 (4\%) pacientes, de quatro a cinco dias em 20 (15\%), e maior do que 5 dias em 112 (81\%). Já no hospital terciário, o tempo de internação foi menor do que três dias em 39 (16\%) pacientes, de quatro a cinco dias em 84 (37\%) pacientes, e maior do que cinco dias em 107 (47\%) pacientes. Por fim, quando avaliada a letalidade dos pacientes no hospital secundário, foram observados 6 (4\%) óbitos, contra 17 (7\%) óbitos do hospital terciário.

Basicamente, no hospital terciário, foi observada frequência significativamente menor, comparando-se ao hospital secundário, no número de pacientes originados na cidade onde o hospital está instalado, no número de indivíduos com mais de 80 anos de idade, no tempo de espera acima de 96 horas, no adiamento da cirurgia por falta de vaga na UTI, no número de procedimentos de raquianestesia, no número de indicações para internação em UTI, no total de complicações, e no tempo de internação acima de 5 dias.

\section{Discussão}

Após as análises, algumas diferenças significantes foram observadas entre os dois hospitais avaliados. A primeira diferença digna de nota referiu-se ao fato de que mais da metade das cirurgias realizadas no hospital secundário ocorreram em pacientes vindos da mesma cidade onde o serviço está localizado. Este resultado contrastou com o quadro verificado no hospital terciário, onde o número de pacientes de cidades diferentes foi praticamente a metade do observado no primeiro hospital. Este fato chama atenção em relação às diferenças nas regiões do estado de São Paulo onde ambos os hospitais estão localizados. O hospital terciário é o Serviço de referência em atendimento a pacientes traumatizados de toda a região de Bragança Paulista, $\mathrm{SP}$, enquanto o hospital secundário, localizado na cidade de Amparo, SP, é um Serviço menos especializado e que recebe pacientes que precisam de atendimento de menor complexidade.

Notou-se uma porcentagem significativamente maior de indivíduos etilistas operados no hospital terciário. A despeito do fato de que tal característica não influenciou nos parâmetros cirúrgicos avaliados na presente pesquisa, a redução da massa óssea em indivíduos que consumem álcool regularmente já foi relatada anteriormente, assim como um aumento da incidência de fraturas em etilistas, fazendo com que o consumo exagerado de bebidas alcoólicas seja um fator contribuinte para a ocorrência de lesões traumáticas (10, 11). Individuos que abusam de álcool têm uma probabilidade quatro vezes maior de sofrer uma fratura do que os indivíduos que não consomem bebida alcoólica pareados pela idade, e boa parte dos indivíduos com traumas ortopédicos estão alcoolizados no primeiro atendimento. Ainda, o álcool é considerado um fator preditivo para não união das extremidades fraturadas, particularmente em pacientes mais jovens com fratura do colo do fêmur (11).

Um dado interessante observado ao final da presente avaliação foi que o número de cirurgias adiadas no hospital terciário por falta de vaga na UTI foi significativamente menor do que no secundário, mesmo este último tendo uma demanda menor de cirurgias. Van Essen et al. (12), ressaltam que o cronograma inicial dos Serviços de cirurgia, muitas vezes, é interrompido pelas cirurgias de emergência, que em sua maioria requerem um suporte avançado no pós-operatório imediato, assim como pela duração prolongada das próprias cirurgias eletivas. Outro fato que pode explicar a maior disponibilidade de leitos de UTI no hospital terciário, foi que a indicação de cuidados intensivos após a cirurgia também foi significativamente menor neste Serviço. Acredita-se que o maior número de pacientes com necessidade de UTI no hospital secundário deva-se ao maior número de remarcações dos procedimentos, conforme descrito, e consequente maior espera pela cirurgia, comentada a seguir.

Realmente um fato marcante identificado neste levantamento foi que o número de pacientes que aguardaram mais de 96 horas para realização da cirurgia foi consideravelmente menor também no hospital terciário. Os fatores relacionados a essa maior espera são os mesmos citados em relação ao maior número de adiamentos, devido à demanda 
por procedimentos cirúrgicos e por vagas na UTI. Parker e Pryor (13) afirmaram que, embora o tempo de espera pela cirurgia não tivesse afetado os indices de mortalidade dos pacientes com fraturas de fêmur proximal por eles operados, assim como observado em nosso estudo, a morbidade indicada pelos autores foi significativamente afetada pelo atraso, particularmente no que diz respeito ao aumento da incidência de lesões por pressão, dentre outras complicações. Todavia, dados mais recentes publicados por Kelly-Pettersson et al. (14), demonstraram que o tempo de espera para a cirurgia está correlacionado com um risco aumentado de mortalidade, assim como de ocorrência de eventos adversos. Ainda, segundo Belmont et al. (15), embora muitas comorbidades pareçam influenciar negativamente no prognóstico de pacientes com fraturas de fêmur, incluindo aumento da morbimortalidade, alguns dos fatores mais significativos dizem respeito à presença de choque, necessidade de diálise, obesidade e tempo de espera para realização da cirurgia. Por fim, Edelmuth et al. (16) relataram que um dos fatores que influencia diretamente no aumento da mortalidade em pacientes com fraturas de fêmur proximal é efetivamente o tempo de espera pela cirurgia.

Seguindo a mesma linha, o número de complicações relacionadas às cirurgias foi significativamente maior no hospital secundário, assim como o tempo de permanência em internação por mais de cinco dias, praticamente o dobro do hospital terciário, provavelmente devido ao maior número de remarcações dos procedimentos, e maior tempo de espera pela cirurgia. Há tempos sabe-se que, quanto antes um paciente é submetido à intervenção cirúrgica, menos tempo este permanecerá no hospital, assim como apresentará menos complicações e melhor reabilitação. Pacientes com fratura proximal de fêmur que aguardam mais de 48 horas pela cirurgia, apresentam risco aumentado de insucesso ou de complicações relacionadas à resolutividade do procedimento $(13,15,14)$.

Os dados aqui observados mostram-se importantes visto que apontam algumas fragilidades no atendimento prestado no hospital secundário. Neste sentido, a despeito de termos trabalhado com uma amostra de conveniência, e acompanhado ambos os Serviços por tempo reduzido, ficam aqui sugestões de melhoria que podem ser utilizadas pelos gestores do hospital secundário na busca pela qualidade no atendimento de pacientes com fraturas de fêmur.

\section{Conclusão}

Embora um maior número de cirurgias tenha sido realizado no hospital terciário, os pacientes atendidos no hospital secundário apresentaram mais complicações, provavelmente em decorrência de mais tempo de espera pelo procedimento, incluindo as cirurgias que precisaram ser remarcadas. É provável também que este fato tenha implicado em maior tempo de internação e de necessidade de encaminhamento para UTI no periodo pós-operatório imediato, fenômenos também observados no hospital secundário.

\section{Notas}

\section{Apoio financeiro}

Este estudo não recebeu apoio financeiro de fontes externas.

\section{Declaração de conflito de interesses}

Os autores declaram não haver conflitos de interesses relevantes ao conteúdo deste estudo.

\section{Contribuições dos autores}

Todos os autores fizeram contribuições substanciais para concepção, ou delineamento, ou aquisição, ou análise ou interpretação de dados; e redação do trabalho ou revisão crítica; e aprovação final da versão para publicação.

\section{Disponibilidade dos dados e responsabilidade pelos resultados}

Todos os autores declaram ter tido total acesso aos dados obtidos e assumem completa responsabilidade pela integridade destes resultados. 


\section{Referências}

1. Jackson C, Tanios M, Ebraheim N. Management of subtrochanteric proximal femur fractures: A Review of Recent Literature. Adv Orthop. 28;2018:1326701. https:// doi.org/10.1155/2018/1326701

2. Daniachi D, Netto A dos S, Ono NK, Guimarães RP, Polesello GC, Honda EK. Epidemiology of fractures of the proximal third of the femur in elderly patients. Rev Bras Ortop. 2015:50(4):371-7. https://doi.org/10.1016/j.rboe.2015.06.007

3. Manninger J, Kazár G. Proximal femur fractures. Definition, epidemiology, anatomy, biomechanics. In: Manninger J, Bosch U, Cserháti P, Fekete K, Kazár $G$ (organizadores). Internal fixation of femoral neck fractures. Vienna: Springer; 2007. p. 1-27. https://doi. org/10.1007/978-3-211-68585-3_1

4. Bittencourt C. Prevenção da osteoporose deve começar na infância, recomenda ortopedista [Internet]. 2015 [citado 2020 Ago 5]. Disponivel em: https://Www. unasus.gov.br/noticia/prevencao-da-osteoporosedeve-comecar-na-infancia-recomenda-ortopedista

5. Soares DS, Mello LM, Silva AS, Martinez EZ, Nunes AA. Fraturas de fêmur em idosos no Brasil: análise espaçotemporal de 2008 a 2012. Cad Saúde Pública. 2014:30:266978. https://doi.org/10.1590/0102-311X00218113.

6. Macedo GG, Teixeira TRG, Ganem G, Daltro GC, Faleiro TB, Rosário DAV, et al. Fraturas do fêmur em idosos: um problema de saúde pública no Brasil. Revista Eletrônica Acervo Científico. 2019;6:e1112. https://doi. org/10.25248/reac.e1112.2019

7. Gallo JJ, Busby-Whitehead J, Rabins PV, Silliman RA, Murphy JB. Reichel Assistência ao Idoso: aspectos clínicos do envelhecimento. 5. ed. Rio de Janeiro: Guanabara Koogan; 2001.

8. Bittar OJNV. Produtividade em hospitais de acordo com alguns indicadores hospitalares. Rev Saúde Pública. 1996;30(1):53-60. https://doi.org/10.1590/ $\underline{\mathrm{S} 0034-89101996000100007}$

9. Doyle DJ, Garmon EH. American Society of Anesthesiologists classification (ASA class). Treasure Island (FL): StatPearls Publishing; 2019.

10. Kristenson H, Redlund-Johnell I. Lower limb fractures and registration for alcoholism. Scandinavian Journal of Social Medicine. 1985;13(3):95-7. https://doi. org/10.1177\%2F140349488501300304

11. Richards CJ, Graf KW, Mashru RP. The Effect of opioids, alcohol, and nonsteroidal anti-inflammatory drugs on fracture union. Orthop Clin North Am. 2017:48(4):433-43. https://doi.org/10.1016/j.ocl.2017.06.002

12. van Essen JT, Hans EW, Hurink JL, Oversberg A. Minimizing the waiting time for emergency surgery. Operations research for health care. 2012;1(2):34-44. https://doi.org/10.1016/j.orhc.2012.05.002

13. Parker M, Pryor $G$. The timing of surgery for proximal femoral fractures. J Bone Joint Surg Br. 1992;74-B(2):2035. https://doi.org/10.1302/0301-620x.74b2.1544952

14. Kelly-Pettersson P, Samuelsson B, Muren O, Unbeck M, Gordon M, Stark A, et al. Waiting time to surgery is correlated with an increased risk of serious adverse events during hospital stay in patients with hip-fracture: A cohort study. Int J Nurs Stud. 2017;69:91-7. https:// doi.org/10.1016/j.ijnurstu.2017.02.003
15. Belmont PJ, Garcia EJ, Romano D, Bader JO, Nelson KJ, Schoenfeld AJ. Risk factors for complications and inhospital mortality following hip fractures: a study using the National Trauma Data Bank. Arch Orthop Trauma Surg. 2014:134(5):597-604. https://doi.org/10.1007/ s00402-014-1959-y

16. Edelmuth SVCL, Sorio GN, Sprovieri FAA, Gali JC, Peron SF. Comorbidades, intercorrências clínicas e fatores associados à mortalidade em pacientes idosos internados por fratura de quadril. Rev bras ortop. 2018:53(5):543-51. https://doi.org/10.1016/i. rboe.2018.07.014

\section{Weby Delsin Mizael}

Médico, especialista em Ortopedia e Traumatologia no Hospital Universitário São Francisco na Providência de Deus (HUSF), em Bragança Paulista, SP, Brasil.

\section{Leandro Campos Vilela}

Médico, especialista em Ortopedia e Traumatologia no Hospital Santa Casa Anna Cintra, em Amparo, SP, Brasil.

\section{Ronaldo Parissi Buainain.}

Médico, especialista em Ortopedia e Traumatologia no Hospital Universitário São Francisco na Providência de Deus (HUSF), em Bragança Paulista, SP, Brasil.

\section{Nilson Nonose}

Médico, especialista em ortopedia e Traumatologia. Preceptor Médico do Serviço de Ortopedia e Traumatologia do Hospital Universitário São Francisco na Providência de Deus (HUSF), em Bragança Paulista, SP, Brasil.

\section{Endereço para correspondência}

Weby Delsin Mizael

Hospital Universitário São Francisco na Providência de Deus

Av. São Francisco de Assis, 260

$12916-542$

Bragança Paulista, SP, Brasil

Os textos deste artigo foram revisados pela Poá Comunicação e submetidos para validação do(s) autor(es) antes da publicação. 